\title{
Presence of Minimal Hepatic Encephalopathy Diagnosed by Computerized Inhibitory Control Test in Patients with Cirrhosis of Liver
}

\author{
Samiullah Shaikh, Kanwal Rai, Waseemullah Shaikh
}

\section{ABSTRACT}

INTRODUCTION: Minimal hepatic encephalopathy is a neuro-cognitive disorder which happens in an epidemic percentage in patients with cirrhosis of liver. The computerized inhibitory control test (ICT) has been proposed as the 'gold standard' test for the diagnosis of MHE by American Association for Study of Liver Diseases (AASLD).

OBJECTIVE: To determine the frequency of minimal hepatic encephalopathy by inhibitory control test (ICT) in patients with cirrhosis of liver.

STUDY DESIGN: Cross sectional study.

PLACE AND DURATION OF STUDY: Medical Unit IV, Liaquat University Hospital, Hyderabad / Jamshoro from 01-12-2012 to 31-05-2013.

METHODOLOGY: The consecutive patients 18 years or more of age, of either gender diagnosed as liver cirrhosis regardless of Child-Pugh class were recruited and evaluated for minimal hepatic encephalopathy through inhibitory control test (ICT). The data was collected on proforma and analyzed in SPSS 16.

RESULTS: Total 110 patients with liver cirrhosis were evaluated for minimal hepatic encephalopathy. The mean age of patients was $46.82 \pm 10.72$. Majority of the subjects were between $30-50$ years of age with male predominance $(74.5 \%)$ and $(47.30 \%)$ were in Child-Pugh class $C(47.3 \%)$. The $>05$ lures/ person and minimal hepatic encephalopathy was identified in 71(64.5\%) patients, of which 60 were male and 11 were female $[P<0.005]$ and majority were in Child Pugh-class $B$.

CONCLUSION: The cirrhotic patients are prone to have minimal hepatic encephalopathy.

KEY WORDS: Cirrhosis, Minimal hepatic encephalopathy, Inhibitory control test.

This article may be cited as: Shaikh S, Rai K, Shaikh W. Presence of Minimal Hepatic Encephalopathy Diagnosed by Computerized Inhibitory Control Test in Patients with Cirrhosis of Liver. J Liaquat Uni Med Health Sci. 2017;16(04):190-3. doi: 10.22442/jlumhs.171640531

\section{INTRODUCTION}

Minimal hepatic encephalopathy (MHE) is a neuro-cognitive disorder which happens in an epidemic percentage in patients with cirrhosis of liver ${ }^{1}$. It is estimated to be present in about $30-80 \%$ of the cirrhotic population'. MHE is a 'misnomer' as it has a huge bearing on the quality of life, capacity to function in daily life and progression to overt hepatic encephalopathy ${ }^{3}$. Moreover MHE is especially dangerous in people who are suffering from it and driving a motor vehicle or operating heavy machinery, so this is no surprise that MHE is invariably linked with motor vehicle accidents all over the world ${ }^{4}$. MHE is usually ignored as a clinical entity by the medical practitioners and gastroenterologists alike. According to American Association for the Study of Liver Diseases $84 \%$ of consultants consider the MHE as significant problem but only $47 \%$ will screen for MHE because psychometric tests are time consuming, difficult to perform and are not standardized ${ }^{5}$. Due to these shortcomings in standardized psychometric testing for the presence of MHE the American Association for Study of Liver Diseases (AASLD) proposed to introduce computerized inhibitory control test (ICT) as the 'alternate gold standard' for the diagnosis of MHE. The ICT is a computerized test of reaction inhibition, attention and working memory, consisting of presentation of several letters at 500 ms periods. It is often validated for the diagnosis and follow through for MHE in the United States (but not in Pakistan) ${ }^{6}$. This test is significantly associated with vehicle crashes and traffic rules violations ${ }^{7}$. The ideal way to diagnose MHE is via combination of EEG (electroencephalogram) and complex psychometric tests. When ICT was compared with these gold standards it identified $88 \%$ of patients suffering from $\mathrm{MHE}^{8}$. The cirrhotic patients are always at risk of accidents while they continue to drive a vehicle ${ }^{9}$. A comprehensive study was conducted by Wein et al ${ }^{10}$ in cirrhotic patients who continued to drive a vehicle. 
The patients were divided in three groups, normal (controls), cirrhotic patients, and patients suffering from MHE. Standardized driving test results showed that the performance of cirrhotic patients was at par with controls $(6 \%$ chance of accidents vs. $8 \%$ in controls), whereas the patients suffering from MHE exhibited $36 \%$ chances of accidents.

The aim of this study is to determine the frequency of minimal encephalopathy with the help of inhibitory control test (ICT) in patients with cirrhosis of liver.

\section{METHODOLOGY}

This cross sectional study included 110 consecutive patients of cirrhosis of liver received at medical OPD, Liaquat University Hospital Hyderabad/Jamshoro from 01-12-2012 to 31-05-2013.

The study was carried out in conformance with the principles of the Declaration of Helsinki. The institutional ethical review committee approved the protocoland consent forms. Written informed permission was taken from all participants of the study.

\section{Sample technique}

Non probability consecutive

Inclusion Criteria

Cirrhosis of liver, male and female gender, adults (more than 18 years of age), All patients had cirrhosis of liver of Child-Pugh class A, B, and C. Patients who were ambulatory and capable of unassisted living (can do household chores and personal hygiene by themselves).

\section{Exclusion Criteria}

Presence of overt hepatic encephalopathy (diagnosed according to West Haven criteria) as these produce false positive cases of minimal hepatic encephalopathy, Mental retardation, sensory or motor deficits, neurological causes of impaired cognition, ongoing systemic illness, electrolyte imbalance, active alcohol or substance abuse (all causes produce false positive cases for minimal hepatic encephalopathy), Illiteracy (those who couldn't use computers or did not know English alphabets.

After taking informed consent Patients fulfilling inclusion criteria were enrolled for the study. All patients gave informed consent. Data recorded on a proforma and relevant laboratory investigations were performed at Liaquat university hospital laboratory. Ultrasound abdomen of patients was done at radiology department of Liaquat University Hospital.

Patients were diagnosed cirrhotic when: Clinical examination detected reduced liver span $(<8 \mathrm{~cm})$ with presence of features of portal hypertension such as ascites and splenomegaly, Biochemically if Prothrombin time was prolonged or serum albumin was $<3.5 \mathrm{~g} / \mathrm{dl}$, and radiologically if the live span was
$<8 \mathrm{~cm}$ in mid-clavicular or portal vein diameter was $>1.3$ or spleen was enlarged in size $(>13 \mathrm{~cm}$ longitudinally). Patients were categorized according to Child-Pugh class.

\section{Computerized inhibitory control test}

Those selected were subjected to computerized ICT. Computerized inhibitory control test program is freely accessible over internet: www.hecme.tv. provided it is used non-commercially, without making any change in the system and the source given due credit by citing.During test all participants were instructed to look at quickly changing letters at the computer screen and pay attention to the $X$ and $Y$ (targets). They were instructed to press "space" when $Y$ follows $X$ or conversely. However in case when they see $\{$ lures $(L)\} X$ is followed closely by another $X$ or $Y$ by another $Y$ need not to press "space". At the end of the test correct and incorrect, incidental key press (correct and wrong reaction to lures, correct estimation and incorrect omission of targets, incidental response) were calculated automatically. Other parameters counted are: total ICT mistake rating (amount of incorrect reaction to lures and wrong omission of targets), target accuracy (TA, percentage between quantity of correctly discovered letter combinations and final amount of proper page combinations) and weighted lures (WL, WL= L/TA2). The presence of $>5$ lures/person was taken as evidence to diagnosed minimal hepatic encephalopathy (MHE) ${ }^{8}$.

\section{Data Analysis Procedure}

Statistical Package for Social Sciences (SPSS) Version 16 was used for data processing. Quantitative variables like age were presented as mean $( \pm S D)$, whereas categorical variables like gender, Child-Pugh classification, age groups, number of lures / person, frequency of minimal hepatic encephalopathy (MHE), were presented as frequencies / proportions. Categorical variables such as gender and Child-Pugh class were compared with presence of minimal hepatic encephalopathy by chi-square test. A $P$ value equal to or less than 0.05 was considered as a significant.

\section{RESULTS}

During six month study period, total 110 patients with liver cirrhosis were evaluated for minimal hepatic encephalopathy. The mean age of patients was 46.82 \pm 10.72 . Among 110 patients $82(74.5 \%)$ were male and $28(25.5 \%$ female. Child-Pugh class $A$ was present in $18(16.4 \%)$ patients, Child-Pugh B in 40 $(36.4 \%)$ and Child-Pugh $C$ in 52(47.3\%). Minimal hepatic encephalopathy with $>5$ lures was present in $71(64.5 \%)$ patients whereas $39(35.5 \%)$ had lures $<5$. Table I shows the demographic characteristics of 
patients included in study. Statistically significant association was found between minimal hepatic encephalopathy and Child-Pugh class $(p=0.000825)$ and gender of the patients $(p=0.001212)$ as MHE was identified in $3 / 18(16.6 \%)$ Child-Pugh class $A, 28 / 40$ $(70 \%)$ class B and $28 / 52(54 \%)$ class C patients; whereas MHE was found in $60 / 82(73.1 \%)$ male patients and $11 / 28(39.2 \%)$ female patients. (Table II).

TABLE I: DEMOGRAPHIC CHARACTERISTICS OF THE PATIENTS $(n=110)$

\begin{tabular}{|l|c|c|}
\hline \multicolumn{1}{|c|}{ Continuous Variables } & Mean & STD \pm \\
\hline Categorical Variables & Frequency & Percentage \\
\hline Male & 82 & 74.5 \\
\hline Female & 28 & 25.5 \\
\hline CHILD-PUGH CLASS & & \\
\hline A & 18 & 16.4 \\
\hline B & 40 & 36.4 \\
\hline C & 52 & 47.3 \\
\hline THE NUMBER OF LURES & & \\
\hline$>5$ & 71 & 64.5 \\
\hline$<5$ & 39 & 35.5 \\
\hline MINIMAL HEPATIC & 71 & 64.5 \\
\hline ENCEPHALOPATHY & - & - \\
\hline
\end{tabular}

TABLE II: RELATIONSHIP OF MINIMAL HEPATIC ENCEPHALOPATHY WITH GENDER AND CHILD-PUGH CLASSIFICATION $(n=110)$

\begin{tabular}{|l|c|c|}
\hline \multicolumn{1}{|c|}{ Variables } & Frequency/Percentage & P value $^{*}$ \\
\cline { 1 - 2 } Male & $60 / 82(73.1 \%)$ & \multirow{2}{*}{0.001212} \\
\hline Female & $11 / 28(39.2 \%)$ & \\
\hline \multicolumn{3}{|c|}{ CHILD - PUGH CLASS } \\
\cline { 1 - 2 } A & $3 / 18(16.6 \%)$ & \multirow{2}{*}{0.000825} \\
\cline { 1 - 2 } B & $28 / 40(70.0 \%)$ & \\
\cline { 1 - 2 } C & $28 / 52(54 \%)$ & \\
\hline
\end{tabular}

${ }^{*}$ Chi square test

\section{DISCUSSION}

In patients with cirrhosis of liver hepatic encephalopathy represents an important marker of survival. As the severity of hepatic encephalopathy increases the survival reduces ${ }^{11}$

Minimal hepatic encephalopathy (MHE) is termed so as there are no any overt signs and symptoms and therefore cannot be identify clinically. The prevalence of $\mathrm{MHE}$ is highly variable ranging from $20-70 \%$, this marked variation may be due to fact that Gold Standard test to diagnosed MHE is yet to be develop ${ }^{12}$. As there is marked cognitive dysfunction in patients with MHE, neuropsychological tests are appropriate to recognize changes in visual perception, attention and concentration.In current study the MHE identified, after positive computerized inhibitory control test results, in71/110 (64.5\%) patients. Jasmohan S. Bajaj et $\mathrm{al}^{8}$, using ICT has reported MHE in $58 \%$ patients with cirrhosis of liver. Das A, Dhiman et al recruited 165 patients with cirrhosis of liver. MHE was observed in 103(62.4\%) patients ${ }^{13}$. Ji-Yao Wang et al in their multicenter study enrolled 538 cirrhotic patients. MHE was observed in 210 (39\%) patients ${ }^{14}$. Sunil Taneja et.al in their study of 102 patients with cirrhosis of liver observed MHE using ICT as tool in 41 $(40.2 \%)^{15}$. The findings of the current study are in very close approximation to those reported Sharma $\mathrm{P}$ et al ${ }^{16}$ and Sadaf Yousuf et $\mathrm{al}^{17}$, who reported it $58 \%$ and $60.7 \%$ respectively. In a case control study comprising 146 healthy volunteers and 53 patients with cirrhosis of liver, Minimal hepatic encephalopathy was observed in $26(49.1 \%)$ cirrhotic patients ${ }^{18}$. MHE is more prevalent when Liver functions decline as high prevalence of MHE has been demonstrated in Child-Pugh class C.In currentstudy we identified MHE in $16.6 \%$ of patients presented in Child-Pugh $\mathrm{A}$, $70.0 \%$ in Child-Pugh $B$ and $54 \%$ cases were in Child-Pugh C.According to Ji-Yao Wang et.al who observed MHE in 207 (39.9\%) out of 519 cirrhotic patients. Among these patients there were $24.8 \%$ in Child-PughA, $39.4 \%$ inChild-Pugh $B$ and $56.1 \%$ in Child-Pugh $\mathrm{C}^{14}$. Das A, Dhiman and colleagues also confirmed in their study that poor liver function is one of important factor in the development of MHE ${ }^{13}$. In another study Sunil Taneja $S$ et al observed MHE in $41(40.2 \%)$ out of 102 cirrhotic patients. Among these $16 / 54$ (29.6\%) were in Child-Pugh class A, 16/35 $(45.7 \%)$ were in Child-Pugh class B and 9/13 (69.2\%) in Child-Pugh class $\mathrm{C}^{15}$. In our study more preponderance of MHE in patients having Child-Pugh $B$ was probably due to disparity in number of patients between Child-Pugh class $B$ and $C$ included in this study.

In this study MHE was present in $73.1 \%(60 / 82)$ of male and $39 \%(11 / 28)$ of female enrolled in the study $(p=0.01)$. According to Jasmohan $S$. Bajaj and colleagues in their 135 patients found a non significant

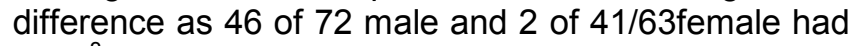
$\mathrm{MHE}^{8}$. 


\section{CONCLUSION}

Keeping in view of the high prevalence of MHE identified by ICT, in patients with cirrhosis of liver, all these patients should really be informed concerning possible risk of progressing to overt encephalopathy as well as to caution against driving.

\section{Limitation of Study}

The ICT to detect MHE can only be performed in patients having knowledge to use computers. Majority of patients with cirrhosis of liver are computer illiterate and therefore deprived of the early detection of MHE.

\section{REFERENCES}

1. Bajaj JS. Minimal hepatic encephalopathy matters in daily life. World J Gastroenterol. 2008; 14 (23):3609-15.

2. Ortiz M, Jacas C, Cordoba J. Minimal hepatic encephalopathy: diagnosis, clinical significance and recommendations. J Hepatol. 2005;42 Suppl:S45-S53.

3. Ferenci $\mathrm{P}$, Lockwood A, Mullen $\mathrm{K}$, Tarter R, Wessenborn K, Blei AT. Hepatic encephalopathy -definition, nomenclature, diagnosis, and quantification: final report of the working party at the 11th World Congresses of Gastroenterology, Vienna, 1998. Hepatology. 2002;35(5):716-21.

4. Mullen K, Ferenci P, Bass NM, Leevy CB, Keeffee EK. An algorithm for the management of Hepatic Encephalopathy. Seminars in Liver Disease. 2007;27:32-48.

5. Bajaj JS, Etemadian A, Hafeezullah M, Saeian K. Testing for minimal hepatic encephalopathy in the United States: an AASLD survey. Hepatology. 2007:45(3):833-4.

6. Bajaj JS, Saeian K, Verber MD, Hischke D, Hoffmann RG, Franco J, et al. Inhibitory control test is a simple method to diagnose minimal hepatic encephalopathy and predict development of overt hepatic encephalopathy. Am J Gastroenterol. 2007;102(4):754-60.

7. Bajaj JS, Saeian K, Schubert CM, Hafeezullah M, Franco J, Varma RR, et al. Minimal hepatic encephalopathy is associated with motor vehicle crashes: the reality beyond the driving test. Hepatology. 2009;50(4):1175-83. doi: 10.1002/ hep.23128
8. Bajaj JS, Hafeezullah M, Franco J, Varma RR, Hoffmann RG, Knox JF, et al. Inhibitory control test for the diagnosis of minimal hepatic encephalopathy. Gastroenterology. 2008;135 (5):1591-1600. doi: 10.1053/j.gastro.2008.07.021.

9. Cordoba J, Lucke R. Driving under the influence of minimal hepatic encephalopathy. Hepatology. 2004;39(3):599-601.

10. Wein C, Koch H, Popp B, Oehler G, Schauder P. Minimal hepatic encephalopathy impairs fitness to drive. Hepatology. 2004;39(3):739-45.

11. Khungar V, Poordad F. Hepatic encephalopathy. Clin Liver Dis.2012;16(2):301-20. doi: 10.1016/ j.cld.2012.03.009.

12. Poordad FF. Review article: the burden of hepatic encephalopathy. Aliment Pharmacol Ther.2007; 25(Suppl):3-9.

13. Das A, Dhiman RK, Saraswat VA, Verma M, Naik SR. Prevalence and natural history of subclinical hepatic encephalopathy in cirrhosis. J Gastroenterol Hepatol. 2001;16(5):531-5.

14. Yu-Yuan Li, Yu-Qiang Nie, Wei-Hong Sha, Zheng Zeng, Fu-Ying Yang, Li Ping, et al. Prevalence of subclinical hepatic encephalopathy in cirrhotic patients in China. World J Gastroenterol.2004;10 (16):2397-2401. doi: 10.3748/wjg.v10.i16.2397

15. Taneja S, Dhiman R, Khatri A, Goval S, Thumbru $\mathrm{K}$, Agarwal R, et al. Inhibitory Control Test for the Detection of Minimal Hepatic Encephalopathy in Patients with Cirrhosis of Liver. J Clin Exp Hepatol. 2012;2(4):306-14. doi: 10.1016/ j.jceh.2012.07.001

16. Sharma $P$, Kumar A, Singh $S$, Tyagi $P$, Kumar $A$. Inhibitory control test, critical flicker frequency, and psychometric tests in the diagnosis of minimal hepatic encephalopathy in cirrhosis. Saudi J Gastroenterol.2013;19(1):40-4.

17. Yousaf S, Farhan S, Nadeem MA, Rasheed S, Mohsin A. Frequency of Minimal Hepatic Encephalopathy in Cirrhotic Patients with Normal Neurological Examination. JMHS 2016; 10(2): 396-98.

18. Li SW, Wang K, Yu YQ, Wang HB, Li YH, Xu JM. Psychometric hepatic encephalopathy score for diagnosis of minimal hepatic encephalopathy in China. World J Gastroenterol 2013; 19(46): 87458751. doi: $10.3748 /$ wjg.v19.i46.8745.

\section{AUTHOR AFFILIATION:}

Dr. Shaikh Samiullah

Associate Professor

Department of Medicine

Liaquat University of Medical \& Health Sciences

(LUMHS), Jamshoro/Hyderabad, Sindh-Pakistan.

Email: samiullahshkh7@gmail.com
Dr. Kanwal Rai

Postgraduate Student

Department of Medicine

LUMHS, Jamshoro, Sindh-Pakistan.
Dr. Shaikh Waseemullah

Department of Anatomy

LUMHS, Jamshoro, Sindh-Pakistan. 